\title{
PESCADORES DE LA TRADICIÓN CULTURAL ENGLEFIELD. DATOS PRELIMINARES EN LA ZONA DEL ESTRECHO DE MAGALLANES Y MAR DE OTWAY XII REGION DE MAGALLANES, CHILE ${ }^{1}$
}

\author{
JIMENA TORRES" Y JIMENA RUZ*
}

\begin{abstract}
RESUMEN
Las primeras ocupaciones de canoeros, 6000 años AP en la zona del estrecho de Magallanes y mar de Otway, cuentan con importante información sobre la caza de mamíferos marinos y su importancia en el modo de vida canoero (Legoupil 1997, San Román 2010, San Román 2011). Igualmente aunque en menor medida, sobre el consumo y explotación de aves (Lefèvre 1997). No obstante hasta el momento nada se sabe sobre la explotación y consumo de peces en esta zona y período en particular. Con el objetivo de conocer este aspecto del modo de vida canoero y la explotación de recursos marinos de ambientes más distantes a la orilla de costa y mayor profundidad, se presentan resultados del estudio ictioarqueológico de la antigua colección arqueológica del sitio Punta Santa Ana 1 recuperada por Omar Ortiz-Troncoso $(1975,1979)$ y atribuido a la tradición cultural Englefield. Así mismo, para comparar ocupaciones del mismo período asumiendo la movilidad de estos grupos y el uso de los diversos microambientes de los mares interiores, se consideran las evidencias de pesca halladas en un sondeo del sitio Pizzulic 2 ubicado en la isla Englefield.

Los resultados muestran una clara orientación a la pesca de Salilota australis (brótula), especie que habita en ambientes de fondo desde el sub-mareal aunque de preferencia a mayores profundidades. En PSA-1 se observa un patrón más diversificado de explotación que en Pizzulic-2. Se destaca la presencia de elementos de Rajiformes (rayas) en ambos sitios especialmente en Punta Santa Ana 1; estos restos por el momento no han aparecido en contextos contemporáneos ni posteriores.
\end{abstract}

PALABRAS CLAVE: estrecho de Magallanes, mar de Otway, modalidad de pesca, tradición cultural Englefield, Salilota australis Rajiformes. 


\title{
PRELIMINARY DATA OF FISHING MODALITIES IN ENGLEFIELD CULTURAL TRADITION (CA. 6000 YEARS) IN THE STRAIT OF MAGELLAN AND OTWAY SEA ZONE, CHILE
}

\begin{abstract}
The first canoe occupations, 6000 yr BP in the Strait of Magellan and Otway sea, count with important information on the hunting of marine mammals and their importance in the canoe way of life (Legoupil 1997, San Román 2010, San Román 2011). Similarly, but to a lesser extent, consumption and use of birds (Lefèvre 1997). Nevertheless, so far nothing is known about the exploitation and consumption of fish in this particular area and period. In order to meet this lifestyle aspect and exploitation of resources from more remote coast environments and marine depths, the results of an ictioarchaeological study of ancient archaeological remains from Punta Santa Ana 1 site, recovered by Omar Ortiz-Troncoso (1975, 1979) and attributed to cultural tradition Englefield, are presented. Also, to compare occupations in the same period, assuming the mobility of these groups and their use of various microenvironments of inland seas, evidence of fish found in a test pit at Pizzulic 2 site, located on the Englefield Island, is considered.

Results show a clear focus on Salilota australis (brótula) fishing, species that lives in seabed environments in sub-tidal areas but prefers greater depths. PSA-1 shows a more diversified pattern of exploitation that Pizzulic-2. The presence of Rajiform elements (rays) in both sites, especially in Punta Santa Ana 1, is highlighted. These remains have not appeared in contemporary or later contexts.
\end{abstract}

KEY WORDS: Strait of Magellan, Otway Sea, fishing modalities, Englefield cultural tradition, Salilota australis, Rajiform.

\section{INTRODUCCIÓN}

En la actualidad la pesca artesanal en el estrecho de Magallanes es una actividad productiva de gran importancia, esto se extiende a lo largo de la zona de mares interiores y archipiélagos patagónicos occidentales hasta la zona de Chiloé. Pese a esto, son escasos los trabajos que han extrapolado este potencial hacia el pasado de las poblaciones prehistóricas (Gaete et al. 2004; Legoupil et al. 2007; Legoupil et al. 2011; Torres 2010; Vargas 2008; Zangrando 2009a-b), esto en proporción a la amplia extensión de territorio ocupado por poblaciones de adaptación marina y considerando su alta movilidad por los fiordos y mares interiores.

En las décadas precedentes, los sitios pertenecientes a los primeros grupos de adaptación marina del estrecho de Magallanes fueron trabajados sin considerar las particularidades de los restos ictioarqueológicos, tanto por las problemáticas de investigación en boga como por el exiguo desarrollo de las metodologías zooarqueológicas en esa época. Al no visualizarse como información relevante del modo de vida, no quedó registro sistemático de este recurso (Emperaire y Laming 1961; Ortiz Troncoso
1975, 1979). No obstante investigaciones posteriores en contextos de tradición cultural Englefield, como los sitios Pizzulic 1 y 2 , han utilizado técnicas de recuperación sistemáticas con el fin de dilucidar su importancia (San Román 2005).

Un dato relevante es que en todos los yacimientos tempranos del área del estrecho de Magallanes y mar de Otway hay referencias sobre la presencia de abundantes restos ictioarqueológicos y de tecnologías asociadas a la pesca como pesos líticos utilizados probablemente para líneas de pesca (tabla 1). En el caso de Bahía Buena en el sector continental del estrecho de Magallanes hay de estas piezas, igualmente aunque en menos cantidad, en el sitio Punta Santa Ana 1 (Ortiz-Troncoso 1979). En el caso de los sitios tempranos de la isla Englefield su presencia es significativa, especialmente en el sitio Bahía Colorada, lo que contrasta con la escasa cantidad de restos de peces posiblemente por preservación. De todas formas en el conjunto de restos de peces se identificó las familias Gadidae y Nototheniidae (Legoupil 1997). En el sitio Englefield 1 también se describen abundantes pesos líticos, presumiblemente usados para la pesca según el autor; además se señala la presencia de abundantes restos 
Tabla 1.

\begin{tabular}{|c|c|c|c|c|c|c|c|}
\hline Sitio & $\begin{array}{c}\text { Estudio } \\
\text { ictioarqueológico }\end{array}$ & Referenc. & $\begin{array}{c}\text { Superficie de } \\
\text { excavación } \\
\text { analizada }\end{array}$ & $\begin{array}{c}\text { Superficie } \\
\text { total de } \\
\text { excavación }\end{array}$ & Ref. del sitio & $\begin{array}{c}\text { Cronología (edad } \\
\text { convencional) }\end{array}$ & $\begin{array}{c}\text { Posibles } \\
\text { útiles para } \\
\text { la pesca }\end{array}$ \\
\hline \multirow{2}{*}{$\begin{array}{c}\text { Englefield } \\
1\end{array}$} & \multirow[t]{2}{*}{ No } & & & & $\begin{array}{c}\text { Emperaire } \\
\text { y Laming } \\
1961\end{array}$ & $\begin{array}{c}8456 \text { et } 9248 \pm \\
1500 \text { años AP }\end{array}$ & \multirow{2}{*}{$\begin{array}{l}\text { Arpón, puntas } \\
\text { multidentadas, } \\
\text { pesos líticos. }\end{array}$} \\
\hline & & & & & Legoupil 1997 & $\begin{array}{l}6100 \pm 110 \\
\text { años AP }\end{array}$ & \\
\hline $\begin{array}{c}\text { B. } \\
\text { Colorada }\end{array}$ & Parcial & $\begin{array}{c}\text { Legoupil } \\
1997\end{array}$ & $\begin{array}{l}\text { Muestra } \\
\text { selectiva }\end{array}$ & & Legoupil 1997 & $\begin{array}{c}5500 \pm 70 \\
\text { años AP }\end{array}$ & $\begin{array}{l}\text { Arpón, puntas } \\
\text { multidentadas, } \\
\text { pesos líticos. }\end{array}$ \\
\hline Pizzulic 1 & $\mathrm{Si}$ & Ruz 2010 & $1 \mathrm{~m}^{2}$ & $1 \mathrm{~m}^{2}$ & $\begin{array}{l}\text { San Román } \\
2005\end{array}$ & $6225+70$ años AP & \\
\hline Pizzulic 2 & $\mathrm{Si}$ & $\begin{array}{c}\text { Torres y } \\
\text { Ruz 2011, } \\
\text { Ruz } 2010\end{array}$ & $1 \mathrm{~m}^{2}$ & $1 \mathrm{~m}^{2}$ & $\begin{array}{l}\text { San Román } \\
2005\end{array}$ & $\begin{array}{l}5945+45 \text { años AP } \\
6055+60 \text { años AP }\end{array}$ & $\begin{array}{c}\text { Arpón, puntas } \\
\text { multidentada, } \\
\text { pesos líticas. }\end{array}$ \\
\hline B. Buena & No & & $\begin{array}{l}\text { Muestra } \\
\text { selectiva }\end{array}$ & $30 \mathrm{~m}^{2}$ & $\begin{array}{c}\text { Ortiz Troncoso } \\
1979\end{array}$ & $\begin{array}{c}\text { 6.849-6.493 } \\
\text { años AP }\end{array}$ & $\begin{array}{c}\text { Arpón, puntas } \\
\text { multidentada, } \\
\text { pesos líticos. }\end{array}$ \\
\hline $\begin{array}{l}\text { P. Santa } \\
\text { Ana } 1\end{array}$ & Parcial & $\begin{array}{c}\text { Torres y } \\
\text { Ruz } 2011\end{array}$ & $\begin{array}{l}\text { Muestra } \\
\text { selectiva }\end{array}$ & $27 \mathrm{~m}^{2}$ & $\begin{array}{l}\text { Ortiz Troncoso } \\
\text { 1979, San } \\
\text { Román et } \\
\text { al. } 2009\end{array}$ & $\begin{array}{c}5620 \pm 120 \\
6410 \pm 70 \text { años AP }\end{array}$ & $\begin{array}{l}\text { Arpón, puntas } \\
\text { multidentada, } \\
\text { pesos líticos. }\end{array}$ \\
\hline
\end{tabular}

de peces (Emperaire y Laming 1961). En tanto el sitio Pizzulic 2 de la misma isla, es el contexto más recientemente estudiado por lo que podemos tener un mejor control de la recuperación de restos de peces, que a su vez también se encuentran asociados a abundantes pesas líticas. Otras evidencias son las puntas óseas multidentadas de Bahía Colorada que pudieron ser utilizadas compuestas en tres piezas en un sólo artefacto a modo de tridente para la pesca, ya que son muy largas y delgadas (Legoupil 1997). Los arpones, en especial los con base cruciforme se vinculan a la caza de mamíferos.

Para el mismo período, en el sur de Tierra del Fuego -al norte del canal Beagle, tres sitios, Túnel 1 (segundo componente), Imiwaia (capa K, L y M) y Mischiuen I (capa F) presentan arpones y pesos líticos muy similares a los mencionados para el estrecho de Magallanes. La actividad de pesca está constatada por abundantes restos de peces, hay en especial orientación a la explotación de Macruronus magellanicus, de la familia Merluccidae (merluzas), la familia Clupeidae (sardinas) y la especie Paranotothenia magallánica, éstas dos últimas especialmente en Túnel 1 (Zangrando 2009a-b). En cambio la especie Salilota australis (brótula) está muy poco representada en estos contextos. Para estas ocupaciones no se descarta la explotación ocasional de merluzas y sardinas en eventos de varamiento masivo estacionales, por lo que no fue un recurso predecible y disponible a lo largo de todo el año (Zangrando 2009a). En contraste, en las ocupaciones más tardías de esta zona hay una mayor intensificación de la actividad de pesca (Juan Muns 1996), expresada en una explotación diversificada de especies litorales, de mayor predictibilidad y abundancia en los contextos. Sumado a esto está la incorporación de la especie Thyrsites atun en el patrón de explotación, lo cual daría además mayor rendimiento (Zangrando 2009a-b).

Al mantener la hipótesis tradicional de un poblamiento inicial de los canales y fiordos meridionales desde los canales patagónicos septentrionales (Legoupil 2005; Rivas et al. 1999) se infiere que tales poblaciones eran conocedoras del mar, la navegación y la explotación de sus recursos marinos. También, es probable que manejasen un conocimiento importante sobre técnicas especializadas de pesca utilizadas desde las embarcaciones, por lo que podrían acceder a peces de ambientes no necesariamente residentes de orilla o que sólo se acerquen en ciertos períodos del año a la orilla. 
Un ejemplo de este conocimiento especializado, justamente en la zona donde comienzan los fiordos y canales patagónicos al norte, es el sitio Piedra Azul frente al seno de Reloncaví. En su segunda ocupación [estrato IV: 5290-5150/5560-5300 AP] hay un predominio de Thrachurus murphys, también son abundantes los restos de Thyrsites atun y de la familia Merluccidae (Gaete et al. 2004, Vargas 2008). Estas mismas características se encuentran en el estrato III, donde además aparecen artefactos para la pesca (Vargas 2008). Si bien en este último estrato no hay fecha, su posición bajo el estrato II de 4410 años y 3960 años AP constituye un bloque cronológico relativamente contemporáneo con los sitios estudiados en este trabajo. La presencia de fauna pelágica y demersal, junto a su abundancia constante en los estratos más tardíos, implicaría cierto conocimiento sobre estrategias de pesca e incluso una orientación selectiva en su captura (Vargas 2008). Esto también se constata en las ocupaciones, aunque más tardías, del sitio Stuven cerca del golfo de Penas (Legoupil et al. 2007).

Este conjunto de datos si bien son escasos en comparación a la inmensa extensión del territorio archipiélagico habitable por los grupos canoeros, nos muestra una solidez en el conocimiento de estas prácticas en las poblaciones de más al norte, lo cual no se debe perder de vista para entender el desarrollo de las poblaciones marinas meridionales si se subentiende que existió un desplazamiento por estas vías marítimas.

De esta manera con el fin de dar un paso más en el conocimiento de la actividad de pesca, su grado de especialización e intensidad en la subsistencia de los grupos de la cultura Englefield, se presenta un avance de los resultados del análisis de peces de los sitios PSA-1 y Pizzulic 2.

\section{ÁREAS Y YACIMIENTOS ESTUDIADOS}

\section{Sector continental: Punta Santa Ana}

Se ubica aproximadamente $50 \mathrm{~km}$ al sur de la ciudad de Punta Arenas, Chile. La punta es el límite norte de la bahía San Juan (figura 1). Presenta una serie de bahías pequeñas a su alrededor propicias para la ocupación humana. El sitio Punta Santa Ana 1 se localiza en la parte media de esta punta en su lado sur, frente a la bahía San Juan, se ubica sobre una terraza de 12 m.s.n.m. (53 37'41" Sur / $70^{\circ} 56^{\prime} 26^{\prime \prime}$ Oeste). El sustrato del emplazamiento es rocoso y presenta una pendiente pronunciada.

La línea de costa actual frente al sitio se encuentra en una franja de roqueríos. Contiguo a este sector hay una pequeña bahía con una playa de cantos y bloques que conforman un conjunto de
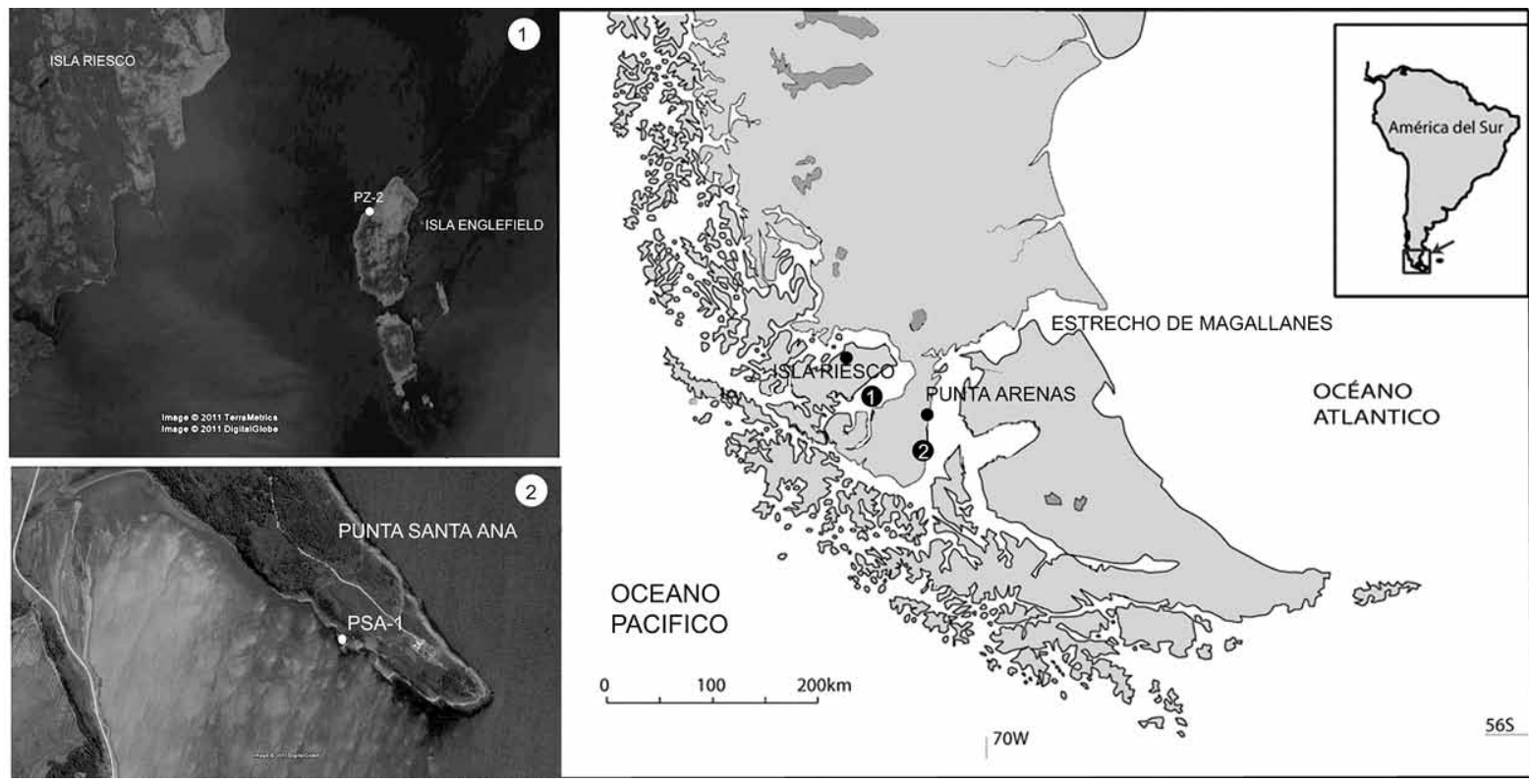

Fig. 1. Área de estudio. 
bermas marinas actuales que también colindan con la ladera abrupta y rocosa donde se sitúa PSA-1.

El sitio Punta Santa Ana 1 (PSA-1) fue excavado por Omar Ortiz Troncoso a principios de la década de los 70' (Ortiz-Troncoso 1979). Se excavó un área total de $29 \mathrm{~m}^{2}$ y el espesor máximo del conchal presentó $55 \mathrm{~cm}$. Los materiales de peces fueron recuperados selectivamente, por lo que su abundancia real pudo ser mucho mayor. En este trabajo hemos analizado la totalidad de restos de peces hallados en la colección del sitio PSA-1 la cual se encuentra en los depósitos de las colecciones arqueológicas del Centro de Estudios del Hombre Austral, Instituto de la Patagonia, Universidad de Magallanes. No todas las unidades presentan restos de peces, no sabemos si se debe a su ausencia real, a un sesgo en la recuperación durante la excavación, o a su extravío en el depósito de colecciones arqueológicas. Aparentemente la colección se encuentra bastante completa, ya que de $29 \mathrm{~m}^{2}$ que se excavaron, hay materiales ictioarqueológicos de 23 unidades de excavación, es decir, de $23 \mathrm{~m}^{2}$, aunque no hay que perder de vista que es recolección selectiva. Los especímenes recuperados corresponden a las 3 capas identificadas por O. Troncoso (1975) ${ }^{4}$.

\section{Sector isla Englefield: Pizzulic 2}

La isla Englefield se encuentra en el mar de Otway el cual está conformado por una entrada de aguas del océano Pacífico. Se sitúa entre la península de Brunswick y la isla Riesco, a $65 \mathrm{~km}$ al oeste de Punta Arenas. El canal Fitz Roy permite que dicho mar se conecte con el de Skyring.

Sobre la costa noroccidental de la isla Englefield, en el marco de prospecciones dirigidas hacia la búsqueda de fuentes de obsidiana verde, se hallaron dos nuevos sitios arqueológicos tempranos, denominados Pizzulic 1 (Pz-1) y Pizzulic 2 (Pz-2), ambos se ubican en una antigua terraza marina a 10 msnm aproximadamente (San Román 2005). En dichos sitios, se encontró una gran cantidad de obsidiana verde y restos de fauna marina como

4 Los materiales arqueológicos fueron rotulados según el criterio de nivel de ocupación. Por ello, se debe realizar la siguiente conversión desde los datos publicados por Ortiz-Troncoso: Nivel IV estratigráfico=Nivel III de ocupación, Nivel III estratigráfico=Nivel II de ocupación, Nivel II estratigráfico=Nivel I de ocupación (San Román 2011). aves, mamíferos marinos y peces. Si bien, ambos sitios son muy cercanos, las dimensiones son dispares, $\mathrm{Pz} 1$ posee un área aproximada de 50 $\mathrm{m}^{2}$ con una potencia estratigráfica máxima de 45 $\mathrm{cm}$. Mientras que, Pz 2 superaría los $250 \mathrm{~m}^{2}$ y 65 $\mathrm{cm}$ de potencial. Una característica de este último contexto es su alta densidad de materiales hallados en un primer sondeo, su riqueza de material está en parte representada por evidencias de obsidiana verde y elementos característicos de la tradición cultural Englefield como una cuña de base cruciforme, una punta ósea multidentada, una base de arpón cruciforme, un pendiente sobre incisivo de otárido y una espátula o cincel sobre ulna de otárido (San Román 2005). Los fechados de $\mathrm{Pz}-2$ provienen del techo de la segunda capa del conchal (5945+45 años AP) y de un lente cercano a la base de la misma capa (6055+-60 años AP).

Para efectos de este trabajo se analizaron los materiales recuperados del primero pozo de sondeo $\left(1 \mathrm{~m}^{2}\right)$ que se hizo en el sitio Pz-2 (San Román 2005, Ruz 2010). Dicha unidad fue excavada por niveles artificiales de $10 \mathrm{~cm}$ y todos los materiales fueron sistemáticamente recuperados y tamizados con una malla de $3 \mathrm{~mm}$, por lo que constituye una muestra confiable.

\section{METODOLOGÍA}

La identificación taxonómica se basó en los elementos esqueletales de la región maxilofaciales (Colley 1990, Wheeler y Jones 1991), también en elementos del neurocráneo como cresta supraoccipital, otolitos y basioccipital. Y por último, en elementos del postcráneo como primera vértebra, vértebras precaudales y en menor medida, vértebras caudales (son menos identificables taxonómicamente en algunos casos).

Para la cuantificación del MNI se seleccionaron las partes esqueletales más abundantes de cada taxón, ya sean individuales (p.e. $1^{\circ}$ vértebra, basioccipital, cresta sagital) o en los pareados el lado más abundante. Para la identificación taxonómica se utilizó una colección osteológica comparativa. En la estimación del NMI (número mínimo de individuos), en cada sitio se consideraron todos los materiales de las distintas unidades de excavación como un solo conjunto.

Para la distribución de talla, en el caso de Genypterus blacodes, Cottoperca gobio y Sebastes 
Tabla 2. Total de restos estudiados.

\begin{tabular}{|c|c|c|c|c|c|c|c|c|}
\hline & \multicolumn{4}{|c|}{ Punta Santa Ana 1 (23 m } & \multicolumn{4}{c|}{ Pizzulic 2 (1 $\left.\mathbf{~ m}^{\mathbf{2}}\right)$} \\
\hline Muestra & NR & NR\% & Peso (gr) & Peso \% & NR & NR\% & Peso (gr) & Peso (\%) \\
\hline NISP & 1234 & 57,57 & 615,1 & 76,67 & 2682 & 66,38 & 540,87 & 80.09 \\
\hline U. anatómicas indent. taxonómicamente & 581 & 27,08 & 242,8 & 21,05 & 250 & 6,18 & 39,34 & 5,82 \\
\hline Fragmentos indet. & 329 & 15.32 & 26,25 & 2,27 & 1108 & 27,42 & 95,85 & 14,19 \\
\hline TOTAL & 2144 & 100 & 884,2 & 100 & 4040 & 100 & 675,26 & 100 \\
\hline
\end{tabular}

oculatus, se ocuparon a lo menos 2 o 3 esqueletos comparativos por especie, para estimar el peso total del individuo (aproximado). En el caso de Salilota australis la distribución de tamaños se basó en las ecuaciones de las regresiones potenciales de elemento esqueletario/longitud estándar y peso según el estudio de alometría de esta especie $e^{5}$. En este trabajo sólo se aplicaron las medidas en basioccipitales y en $1^{\circ}$ vértebras, ya que son las piezas más abundantes del conjunto. Por ultimo, se pesó el material (gr.) en una pesa de 3 dígitos.

\section{ESPECIES EXPLOTADAS EN LOS SITIOS PUNTA SANTA ANA 1 Y PIZZULIC 2}

En PSA-1 hay 9 taxones, no obstante se observa un predominio de restos de Salilota australis (brótula), especie bentodemersal, que supera la mitad de la muestra estudiada (tabla 3, figura 2). Se pudo estimar que las tallas de los ejemplares de Salilota australis se encuentran preferentemente entre 46 y $55 \mathrm{~cm}$ de longitud estándar (LS) y un peso entre $1 \mathrm{~kg}$ y $2 \mathrm{~kg}$ (figura 3). No obstante, hay un amplio rango desde tallas pequeñas como $36 \mathrm{~cm}$ que puede aproximarse a 550 gr hasta individuos de $74 \mathrm{~cm}$ de LS que equivale aproximadamente a 4 $\mathrm{kg}$. Estas medidas coinciden con las tallas máximas señaladas en estudios para este taxón en el océano atlántico (Pérez Comas 1980; Cassia y Hansen, 2005, Cassia 2006). En la actualidad, se han colectado en el estrecho de Magallanes cerca de 70 ejemplares de esta especie, en distintos meses del año para una colección de estacionalidad. Según la información obtenida la talla máxima es de $2 \mathrm{~kg}$ aproximadamente con una longitud estándar de 58 $\mathrm{cm}$, lo cual no corresponde con nuestras evidencias arqueológicas. Esto podría explicarse debido a

5 El estudio de alometría de la especie Salilota australis es parte de los estudios desarrollados en el marco del doctorado efectuado por la autora principal de este trabajo. El trabajo se encuentra en desarrollo. que los individuos adultos de mayor edad y talla se encuentran a mayores profundidades. Una ventaja es que esta especie aún se encuentra escasamente comercializada y por tanto no ha estado expuesta a una presión pesquera tan intensa como en otros taxones, por ejemplo, las merluzas que hoy en día se encuentran sobreexplotadas. De esta manera, los estudios de dinámica poblacional actual puede corresponder mejor a su dinámica en el pasado, aunque los cambios ambientales ocurridos en 6000 años pudieron influir en su comportamiento. Es de esperar que los grupos canoeros utilizasen técnicas de pesca desde sus embarcaciones o a orilla de costa, aunque de todos modos no a profundidades como en la pesca actual de esta especie (artesanal o industrial). También es posible que hayan aprovechado los desplazamientos verticales de esta especie entre el día (mayor profundidad) y la noche (suben a menores profundidades). Una hipótesis es que en el pasado los individuos adultos de mayor tamaño se acercaban más a ambientes de menor profundidad por factores que desconocemos. Se debe tomar en cuenta que en la actualidad esta especie vive a grandes profundidades (hasta 900 o $1000 \mathrm{~m}$ en el talud) de preferencia entre 50 y 300 metros (Wöhler et al. 2004, Cassia 2006); aunque ha sido vista ocasionalmente a 10 m de profundidad (Lloris y Rucabado 1991) y en el sublitoral somero, hasta $35 \mathrm{~m}$ (Sielfeld et al. 2006). Particularmente son los juveniles los que se acercan a estas profundidades (Cassia 2006).

El resto de taxones presenta un bajo porcentaje. No obstante el \% de NISP de las rayas (orden: Rajiformes) es alto aunque representado sólo por vértebras, por lo que podría disminuir bastante su representación del MNI. Es novedosa la presencia de este taxón, ya que en ninguno otro sitio canoero de Patagonia han aparecido restos. El ambiente donde habitan estos peces cartilaginosos, es esencialmente bentónicos de fondo arenoso o fangoso, por lo que independiente que por ahora no se logre una mejor resolución de su taxonómica a nivel de especie o 
Tabla 3. Diversidad taxonómica del conjunto de PSA-1 y PZ-2.

\begin{tabular}{|l|r|r|r|r|r|r|r|r|r|r|}
\hline \multicolumn{1}{|c|}{ Taxón } & \multicolumn{9}{|c|}{ PSA-1 } & \multicolumn{5}{|c|}{ PZ-2 } \\
\hline & NISP & $\begin{array}{c}\text { NISP } \\
\text { \% }\end{array}$ & MNI & $\begin{array}{c}\text { MNI } \\
\text { \% }\end{array}$ & $\begin{array}{l}\text { Peso } \\
\text { (grs) }\end{array}$ & NISP & $\begin{array}{c}\text { NISP } \\
\text { \% }\end{array}$ & MNI & $\begin{array}{c}\text { MNI } \\
\text { \% }\end{array}$ & $\begin{array}{c}\text { Peso } \\
\text { (grs) }\end{array}$ \\
\hline Austrolycus sp. (morena) & 18 & 1,45 & 3 & 3,7 & 22 & 5 & 0,18 & 2 & 2,32 & 3,05 \\
\hline Cottoperca gobio (toro de los canales) & 32 & 2,59 & 4 & 4,93 & 7,3 & 289 & 10,77 & 16 & 18,6 & 60,47 \\
\hline Eleginops maclovinus (róbalo) & 60 & 4,86 & 6 & 7,4 & 38,8 & 19 & 0,7 & 1 & 1,16 & 47,66 \\
\hline Genypterus sp. (congrio) & 114 & 9,23 & 7 & 8,64 & 112 & 64 & 2,38 & 4 & 4,65 & 3,65 \\
\hline Macruronus magellanicus (merluza de cola) & 65 & 5,26 & 6 & 7,4 & 30,8 & 0 & 0 & 0 & 0 & 0 \\
\hline Merluccius sp. (merluza común o austral) & 2 & 0,16 & 1 & 1,23 & 0,4 & 0 & 0 & 0 & 0 & 0 \\
\hline Rajiformes (raya) & 218 & 17,66 & $?$ & 52,95 & 9 & 0,33 & 1 & 1,16 & 0,8 \\
\hline Salilota australis (brótula) & $\mathbf{6 8 9}$ & $\mathbf{5 5 , 8 3}$ & $\mathbf{5 0}$ & $\mathbf{6 1 , 7 2}$ & 337 & 2296 & 85,6 & 62 & 72,09 & 425,2 \\
\hline Sebastes oculatus (cabrilla) & 36 & 2,91 & 4 & 4,93 & 13,85 & 0 & 0 & 0 & 0 & 0 \\
\hline Total & $\mathbf{1 2 3 4}$ & $\mathbf{1 0 0}$ & $\mathbf{8 1}$ & $\mathbf{1 0 0}$ & $\mathbf{6 1 5 , 1}$ & 2682 & 100 & 86 & 100 & 540,8 \\
\hline
\end{tabular}

de género, su presencia implica necesariamente la captura en estos ambientes. Hay especies de rayas de profundidad y otras costeras, por lo que las encontradas en el contexto pudieron ser capturadas tanto en aguas someras en la orilla de costa, como desde alguna embarcación a mayor profundidad. Al no tener certeza de su clasificación taxonómica específica, lo hemos determinado genéricamente a nivel del suborden Batoidea. No obstante, probablemente pertenezcan a la familia Pseudorajidae debido a su abundancia en estas latitudes (Lamilla y Sáez 2003; Venegas y Sielfeld 1998; Sánchez y Mabragaña 2002) y por la similitud de las vértebras del esqueleto de referencia de un ejemplar de esta familia.

Ya en un porcentaje bastante más bajo se encuentra Genypterus blacodes (congrio), es una especie bentónica demersal común en esta zona biogeográfica, que pudo ser capturada junto a la brótula como fauna acompañante. Los ejemplares tienen entre $2 \mathrm{~kg}$ y $10 \mathrm{~kg}$; Por lo general los ejemplares de mayor tamaño son capturados en zonas de mayor profundidad y los juveniles se aproximan a aguas someras pudiendo ser capturados desde la orilla.

Los restos de especies de merluzas también son escasos, como Macruronus magellanicus (merluza de cola) y Merluccius sp., (merluza austral); ellas pudieron ser capturadas también como fauna acompañante de Salilota australis, al igual que Sebastes oculatus (cabrilla común), aunque este último también frecuenta orillas rocosas donde pudo capturarse junto a Astrolycus sp. y Cottoperca gobio. La cabrilla presenta una estimación de peso

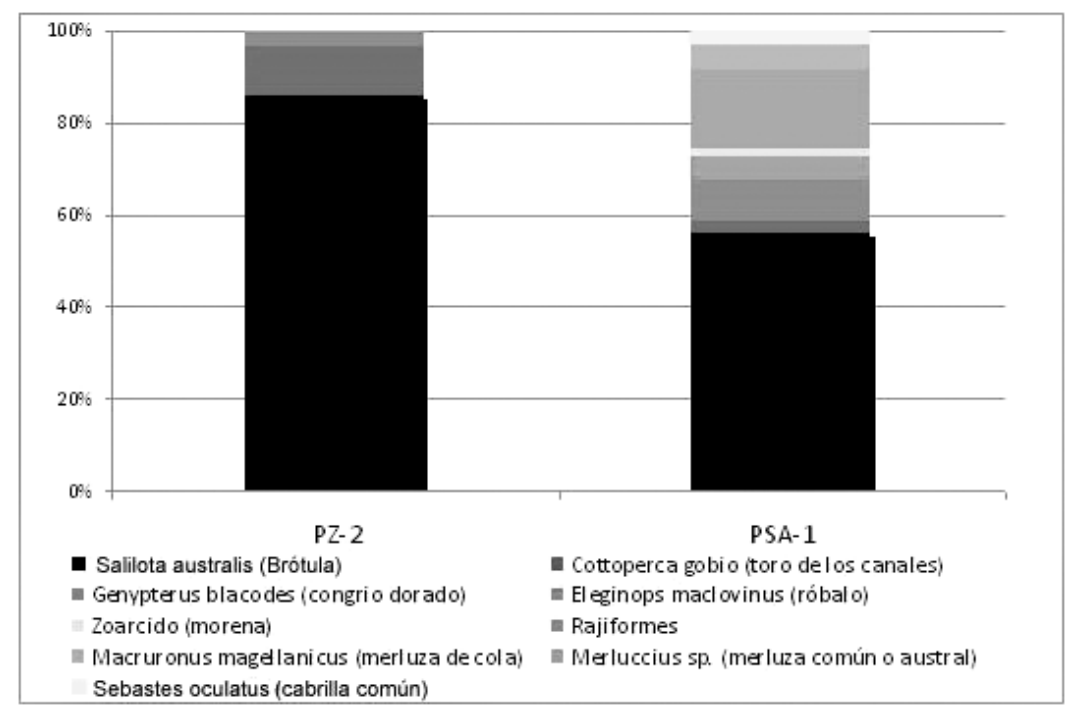

Fig. 2. Diversidad taxonómica sitio PSA-1 y PZ-2 (NISP \%). 
entre 800 gr y $2 \mathrm{~kg}$, aunque la mayor parte de elementos (MNI) presenta entre $1 \mathrm{~kg}$ y $1,5 \mathrm{~kg}$ que son su peso promedio.

Las demás especies propias de ambientes de orilla, presentan exiguos restos como Eleginops maclovinus (róbalo), Austrolycus sp. (Morena) y Cottoperca gobio (torito de los canales). La primera frecuenta aguas someras y sectores de desembocaduras, mientras que las demás son de sectores de intermareal o submareal rocosos. En el caso del torito de los canales hay individuos entre 350 gr y $1,5 \mathrm{~kg}$.

Las morenas (zoárcidos) son una especie muy común en el intermareal rocoso y aguas someras de no más $5 \mathrm{~m}$ de profundidad.

En la actualidad el ambiente intermareal y submareal rocoso de punta Santa Ana, posee concentraciones de algas y fauna malacológica posibles de recolectar en baja marea. Pescadores locales señalan que el róbalo sale en las redes. Según estudios en curso sobre la biodiversidad estacional del ambiente rocoso asociado a bosques de algas (Macrosystis pyrifera) en la punta Santa Ana, las especies que más aparecen son Patagonotothen sp., especialmente la especie cournucola, la especie $C$. gobio y zoarcidos como Austrolycus sp. (com. pers. César Cárdenas y Emma Newcombe 2010). Todas estas, menos Patagonothothen sp., aparecen en el sitio aunque en escasa frecuencia.

En cuanto a la distribución estratigráfica, la capa 2 es la que presenta mayor concentración y diversidad taxonómica, en cambio en la capa 1 ( $1^{\circ}$ ocupación) y 3 ( $3^{\circ}$ ocupación o superior) son escasos (figura 4). Sólo en la capa 2 aparecen Austrolycus sp., Cottoperca gobio y Merluccius sp.

En el caso de Pizzulic 2, hay menos diversidad de especies explotadas, 6 taxones, y se acentúa aún más la importancia de la brótula, este predominio se encuentra representado tanto por la cantidad de restos como por su porcentaje en relación a los demás taxones, con un NISP de 85,6\% (tabla 2). Esta proporción se mantiene constante a lo largo de toda la estratigrafía, aunque se concentra en las capas intermedias, especialmente en el nivel 5 (figura 5). En este contexto no hay cabrilla ni algún tipo de

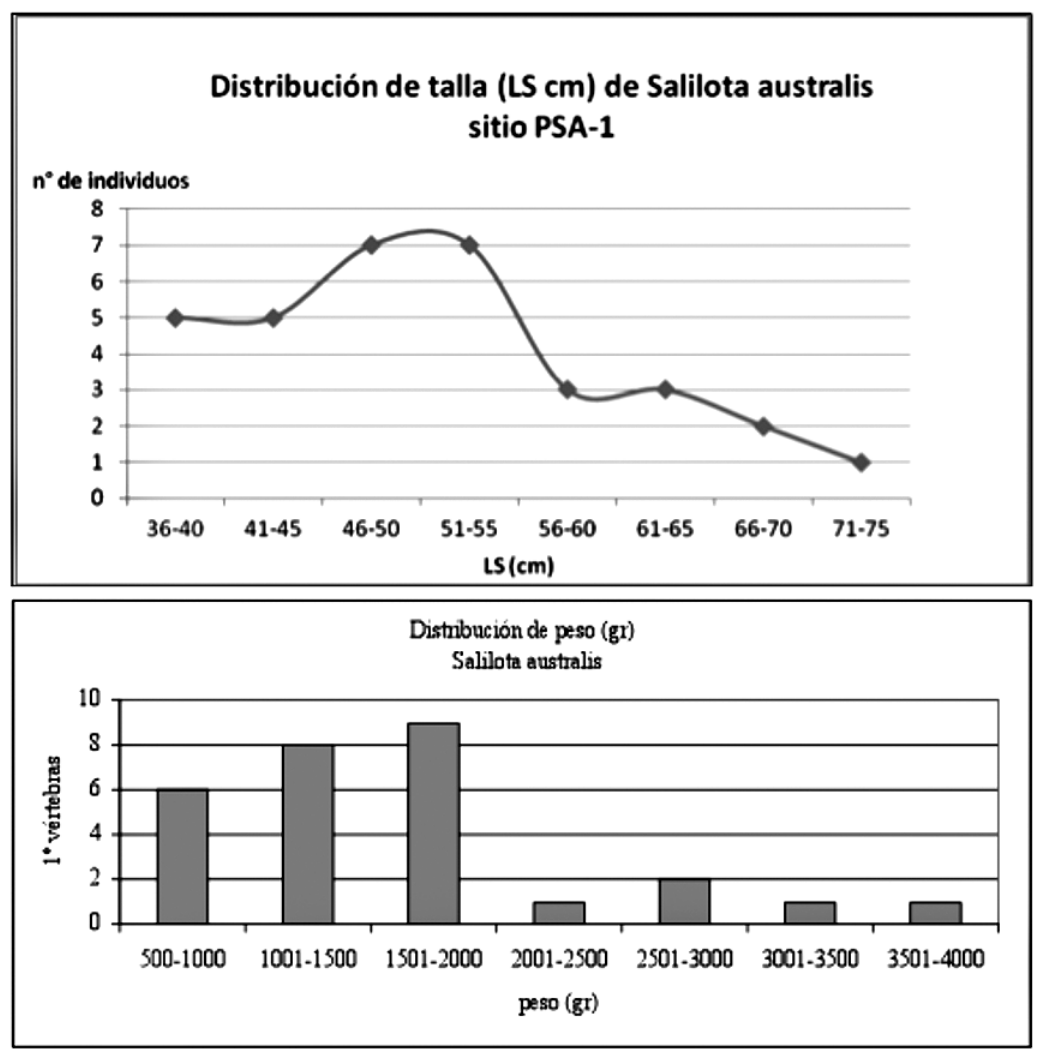

Fig. 3. Distribución de talla y peso de Salilota australis, Sitio PSA-1. 


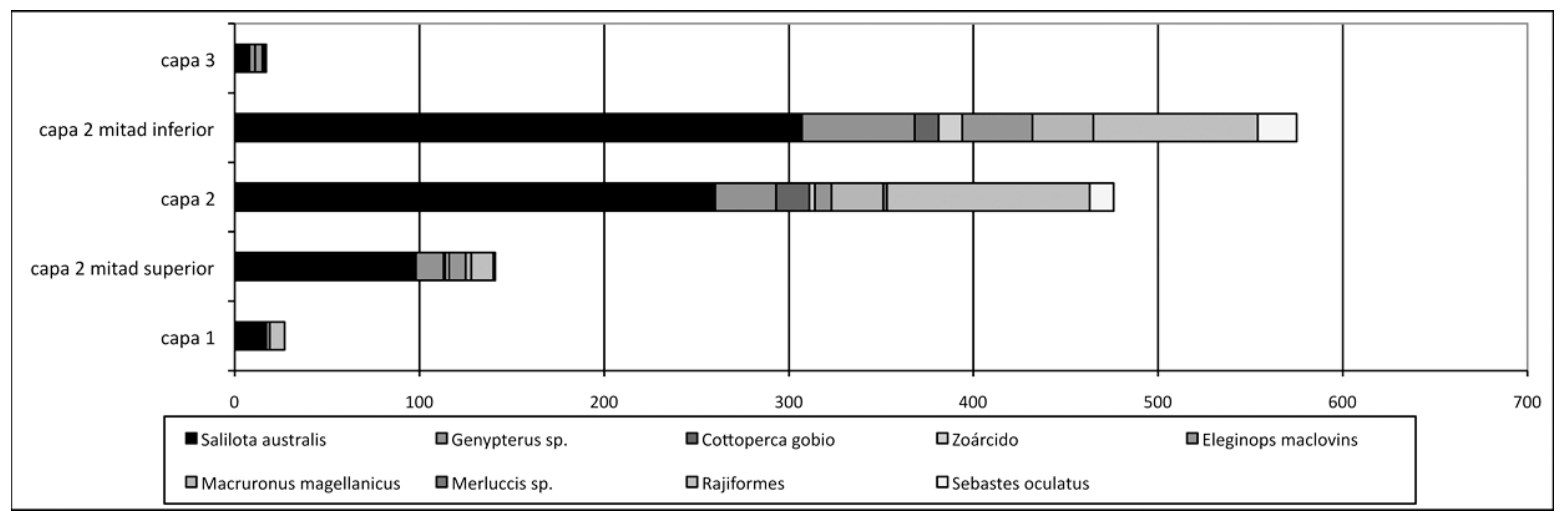

Fig. 4. Sitio PSA-1. Diversidad taxonómica y distribución por capa (NISP).

merluza, sólo restos de congrio dorado. Sus restos se encuentran casi en todos los estratos, salvo en el nivel 8 y 10 , mientras que la mayor concentración esta en el nivel 7 disminuyendo hacia las capas superficiales.

Sobre los peces de ambientes de orilla, es importante la presencia del torito de los canales, es la segunda especie más representada con un $10,77 \%$ del NISP, esta acompaña a la brótula en toda la secuencia y en términos generales se comporta del mismo modo que las otras especies, es decir, con una mayor cantidad en los estratos medios, pese a que muestra un leve aumento hacia los estratos más profundos. Los restos de Eleginops maclovinus alcanzan un NISP\% de sólo $0,18 \%$ y esta representado casi sólo por vértebras (NISP 16), la mayor cantidad se encuentra en la capa 7 (NISP 6). El MNI es igualmente bajo, de sólo 1 individuo. De la familia de los Zoárcidos posiblemente Austrolycus depressiceps (morena) fueron identificados 5 fragmentos, todos ellos huesos maxilofaciales presentes en los niveles 3,6 y 7 .

\section{DISCUSIÓN}

En ambas ocupaciones adscritas a los primeros grupos de adaptación marina de la zona de estudio, se distingue claramente un énfasis en la pesca de la especie Salilota australis (brótula). Esta orientación también se distingue en ocupaciones de 2500-3500 años AP en la misma zona (Torres 2010, Ruz 2010, Legoupil 2011). Esto contrasta con la casi ausencia en los sitios de canoeros tempranos del más al sur (norte canal Beagle), como también la escasa importancia actual que se le da a su explotación artesanal $e$ industrial en la zona del estrecho de Magallanes y en general en la vertiente pacífica.

Si bien esta orientación a la pesca de brótula es característica de ambos sitios estudiados, también se pueden observar diferencias en el patrón de explotación posiblemente consecuencia de las diferencias ambientales: en el caso de $\mathrm{Pz}-2$, una isla con escasos afloramientos de agua dulce (hoy en día

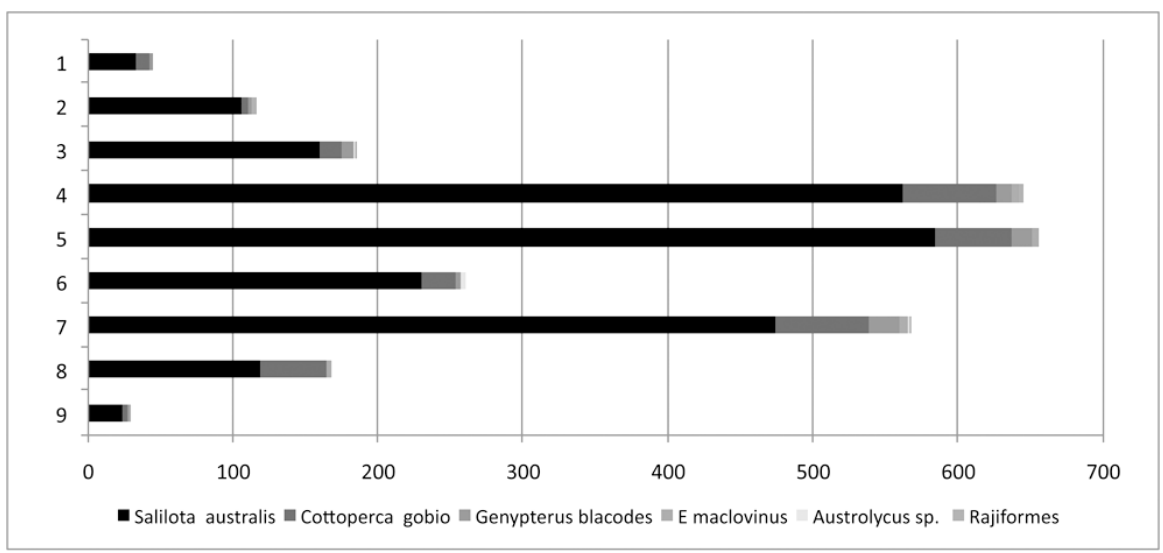

Fig. 5. PZ-2, diversidad taxonómica (NISP) y distribución por nivel artificial de excavación. 
sólo hay vertientes secas), y en el caso de PSA-1, una península próxima a causes de agua dulce. De esta manera, en PSA-1 se observa una actividad de pesca más diversificada, no sólo por el número de taxones y su representación, sino también por la captura de peces de distintos microambientes como: ambiente fangoso o arenoso (rayas), ambientes cercanos a desembocaduras (róbalo); zonas de intermareal o submareal rocoso (torito de los canales y morenas), mientras que la pesca de merluza austral pudo ser en los mismos eventos de pesca de brótulas y congrios. Lamentablemente no sabemos con certeza si hay pesca de especies de tamaño pequeño como sardinas (Clupeidae), pejerreyes o nototénidos como Patagonotothen sp., ya que en el marco de una recolección selectiva, pudieron no ser recolectadas por su tamaño. De todos modos, la presencia de peces cartilaginosos como las rayas dan cuenta de la buena preservación del contexto, por lo cual, si hubo consumo de estas especies pequeñas tuvieron que estar presentes en el contexto arqueológico al momento de la excavación. Un nuevo análisis sobre una muestra bien controlada del sitio permitirá saber con mayor claridad el espectro real de especies consumidas. Indirectamente por medio de la cuantificación de MNI de Salilota australis podemos desprender que la cantidad de restos presentes en el sitio PSA-1 pudo ser mucho mayor a lo recuperado, ya que si bien se encontraron sólo 689 restos de esta especie, se cuantificaron 50 basioccipitales, lo cual es casi equivalente al número de basioccipitales de Pz-2 (53 de estas piezas) sólo que el total de restos de este taxón son 2.296 en 1 $\mathrm{m}^{2}$. Este simple cálculo da cuenta de la densidad de restos del sondeo de $\mathrm{Pz}-2$ y también la selección de restos recuperados en PSA- 1 que pudo subestimar la estimación de su importancia.

En cambio en el caso de $\mathrm{Pz}-2$, hubo una exhaustiva recuperación del material ictioarqueológico. Aún así hay menor diversidad de especies que en PSA-1 y de todas formas, la brótula es la especie más explotada. El torito de los canales también mostró una buena representación, de lo que se deduce la explotación de peces del ambiente rocoso intermareal o submareal. En este sitio no se hallaron ni merluza de cola ni merluza austral, tampoco la presencia de taxones pequeños como sardinas, pejerreyes o nototénidos como Patagonotothen sp. Considerando la rigurosidad de la recolección de material ictioarqueológico, es más seguro que estas especies no se encuentran representadas por lo que descartamos su consumo. La ausencia de sardinas y merluzas de cola en $\mathrm{Pz}-2$ disminuye la posibilidad del aprovechamiento de varamientos estacionales de estas especies, como ha sido bien registrado en zonas del canal Beagle y también en algunas zonas del estrecho de Magallanes. De esta manera se concluye que las especies mejor consumidas de ambos sitios son de ambientes preferentemente de mayor profundidad, aunque en $\mathrm{Pz}-2$ se puede observar mayor selectividad y especialización en sólo dos taxones ya que prevalece la brótula y el torito de los canales, ambos de ambientes diferentes.

Es posible señalar que en este primer período de pescadores recolectores cazadores en los archipiélagos meridionales, hay diferencias en el patrón de explotación de peces entre la zona del estrecho de Magallanes- mar de Otway, con el de las ocupaciones del norte del canal Beagle. En los sitios tempranos del norte del canal Beagle, la especie predominante es Macruronus magellanicus, la familia Clupeidae y el género Merluccius sp, en cambio la Salilota australis está muy poco representada (Zangrando 2009a-b). Para estas primeras ocupaciones no se descarta la explotación ocasional de estas especies en eventos estacionales de varamiento masivo, por lo cual no fue un recurso predecible y disponible a lo largo de todo el año (Zangrando 2009b). En la actualidad se han observado grandes varamientos, tanto de merluza de cola como de sardina en la estación estival. En distintas zonas se han reportado, como canal Beagle, Puerto Arturo en la costa occidental de Tierra del Fuego, punta Carrera en el estrecho de Magallanes, entre otras localidades. No obstante estos eventos no han sido vistos integrados por la especie Salilota australis.

No sabemos por lo pronto si la ausencia de Salilota australis en sitios del canal, se debe a un patrón cultural o a diferencias en la distribución y abundancia biogeográfica en ese período del Holoceno medio. No obstante, en la actualidad se encuentra disponible tanto en el canal Beagle como en el estrecho de Magallanes, aunque en el caso del canal Beagle, los pescadores deben distanciarse más de la costa para pescarla.

Respecto a las tecnologías de pesca, en los sitios tempranos del estrecho de Magallanes destaca la abundancia de pesas líticas posiblemente para la pesca, así como la presencia de puntas multidentadas, estos elementos están en todos los sitios (tabla 1). 
Sobre la funcionalidad exacta, de las pesas, si bien es difícil saberlo con total seguridad, hay mayores argumentos de su uso como pesas de línea de pesca; esto principalmente por la información etnográfica sobre las características de estas piezas y su uso por los grupos yámanas (Orquera y Piana 1999, Martial et al. 2007). Como se mencionó, estas piezas son propias de los componentes tempranos del estrecho de Magallanes, son especialmente abundantes en el sitio bahía Colorada en isla Englefield (Legoupil 1997). También en las excavaciones realizadas en Pizzulic 2, han aparecido abundantes pesos líticos de similares características.

De esta manera, la presencia y abundancia de tecnologías especializadas en todos los sitios, sumado a la pesca orientada a individuos de gran talla, que pese a los cambios ambientales ocurridos en 6000 años, es probable que los adultos grandes no se hayan encontrado tan accesibles para la pesca como los adultos más pequeños o juveniles; nos incita a pensar que los ocupantes de estos sitios ya contaban con conocimientos especializados sólidos en la explotación de estas especie bentodemersal.

Para finalizar, si bien hay elementos que muestran una vinculación cultural entre las ocupaciones del canal Beagle y el Estrecho de Magallanes en este período, es necesario estudiar en profundidad otras zonas y distintos ambientes ocupados por estos grupos canoeros que se desplazaban por los mares interiores explorando diferentes ambientes. De esta manera, para hablar y aproximarnos con datos consistentes a su patrón de movilidad, uso del territorio y la riqueza de sus recursos, no queda más que asumir una escala espacial mayor y considerar los factores estacionales en la movilidad. Bajo esta línea se pretende seguir trabajando en lo que concierne a las estrategias de subsistencia y estacionalidad.

\section{AGRADECIMIENTOS}

Agradecemos a Philippe Béarez investigador del UMR 7209-CNRS (Francia), por atender a nuestras consultas y a Pedro Cárdenas, conservador del Centro de Estudios del Hombre Austral, Universidad de Magallanes, el cual participó en la primera excavación de PSA-1 junto al arqueólogo Omar Ortiz -Troncoso, y quién nos permitió acceder a la colecciones y brindó información relevante sobre el proceso de excavación y recuperación.

\section{BIBLIOGRAFÍA}

CASSIA M.C. Y J. HANSEN 2005. Distribución estacional y estructura poblacional del bacalao austral (Pisces, Moridae, Salilota australis) en la zona económica exclusiva Argentina entre los $45^{\circ} \mathrm{S}$ y $56^{\circ} \mathrm{S}$ durante 1992 y 1993. Revista de Investigación de Desarrollo Pesquero 17: 55-65.

CASSIA M. C. 2006. Distribución y estructura poblacional de tres especies de peces pertenecientes al área austral: Micromesistius australis, Salilota australis y Dissostichus eleginoides. Tesis Doctoral. Facultad de Ciencias Exactas y Naturales. Universidad Nacional de Mar del Plata, 170p. MS

COLLEY, S. 1990. The analysis and interpretation of archaeological fish remains. En: Advances in Archaeological Method and Theory, Vol.2. M. B Shiffer (ed), pp.207253. Academic Press, San Diego.

EMPERAIRE J. Y A. LAMING 1961. Les gisements des iles Englefield et Vivian. Journal de la Société des Américanistes 50: 7-75.

GAETE, N., X. NAVARRO, F. CONSTANTINESCU, C. MERA, D. SELLES, M. E. SOLARI, M. L. VARGAS, D. OLIVA Y L. DURÁN 2004. Una mirada al modo de vida canoero del mar interior desde Piedra Azul. Chungará 36:333-346.

JUAN-MUNS, N. 1996. Aprovechamiento de los recursos ícticos en Túnel VII (Tierra del Fuego). En: Arqueología Sólo Patagonia. Actas de las Segundas Jornadas de Arqueología de Patagonia. Pp: 89-98. Centro Nacional Patagónico, CONICET, Puerto Madryn.

LAMILLA, J. Y S. SÁEZ 2003. Clave taxonómica para el reconocimiento de especies de Rayas chilenas (Chondrichthyes, Batoidei). Investigaciones Marinas, Valparaíso, 31(2): 3-16.

LEFÈVRE C. 1997. Les Oiseaux. Chapitre 4. En: Bahia Colorada (île d'Englefield). Les premiers chasseurs de mammifères marins de Patagonie australe, D. Legoupil compiladora. Memoires de l'A.D.P.F., Recherches sur les Civilisations, Paris.

LEGOUPIL D. 1997. Bahia Colorada (île d'Englefield). Les premiers chasseurs de mammifères marins de Patagonie australe. Memoires de l'A.D.P.F., Recherches sur les Civilisations, Paris.

2005. Recolectores de mariscos tempranos en el sureste de la isla de Chiloé. Magallania 33(1):51-61.

LEGOUPIL, D., P. BEAREZ, S. LEPETZ Y M. SAN ROMÁN. 2007. De la pesca a la caza: evolución económica del sitio Stuven 1, al sur del Golfo de Penas. En: Arqueología de 
Fuego Patagonia, levantando piedras, desenterrando huesos, develando arcanos. Editado por F. Morello, M. Martinic, A. Prieto, G. Bahamondes. Ediciones CEQUA, Punta Arenas.

LEGOUPIL D., P. BÉAREZ, C. LEFÈVRE, M. SAN ROMÁN, J. TORRES. 2011. Estrategias de aprovisionamiento de cazadores recolectores de isla Dawson (estrecho de Magallanes) durante la segunda mitad del Holoceno: primeras aproximaciones. Magallania 39(2):153-164.

LLORIS, D Y J. RUCABADO 1991. Ictiofauna del Canal de Beagle (Tierra del Fuego). Aspectos ecológicos y análisis biogeográfico. Publicaciones Especiales del Instituto Español de Oceanografía, Madrid.

MARTIAL, L.F., J. DENIKER Y P. HYADES 2007. Etnografía de los Indios Yaghan. En: La Misión Científica del Cabo de Hornos 1882-1883. D. Legoupil y A. Prieto editores científicos. Ediciones Universidad de Magallanes-Instituto Francés de Estudios Andinos, Punta Arenas.

ORQUERA, L. Y E. PIANA. 1999a. La vida material y social de los Yámana. EUDEBA, Buenos Aires.

1999b. Arqueología de la Región del Canal Beagle (Tierra del Fuego, República Argentina). Sociedad Argentina de Antropología, Buenos Aires.

ORTIZ-TRONCOSO, O. 1975. Los yacimientos de Punta Santa Ana y Bahía Buena (Patagonia Austral): Excavaciones y fechados radiocarbónicos. Anales del Instituto de la Patagonia 6: 93-122.

1979. Punta Santa Ana et Bahia Buena: deux gisements sur une ancienne ligne de rivage dans le Détroit de Magellan. Journal de la Société des Américanistes 56: $133-204$.

PEREZ COMAS, J. 1980. Distribución, aéreas de concentración y estructura de la población del Bacalao austral (Salilota australis, Gunther, 1887) del Atlántico sudoccidental. Revista de investigación y desarrollo pesquero 2(2):22-37.

RIVAS P., C. OCAMPO Y E. ASPILLAGA 1999. Poblamiento temprano de los canales patagónicos: El núcleo ecotonal Septentrional. Anales del Instituto de la Patagonia, Serie Ciencias Humanas 27:221-230.

RUZ, J. 2010. Informe de práctica Análisis Ictioarqueológico de los sitios Pizzulic 1, 2 y 3. Practica profesional. Departamento de Antropología, Facultad de Ciencias Sociales, Universidad de Chile. MS.

SÁNCHEZ M. F. Y E. MABRAGAÑA. Características biológicas de algunas Rayas de la región Subpatagónica. INDEP informe técnico $\mathrm{N}^{\circ} 48$, noviembre 2002. Argentina.

SAN ROMAN, M. 2005. Nuevos hallazgos de sitios de cazadores recolectores marinos tempranos en isla Englefield, mar de Otway. Magallania 33(2):173-176.
2010. La explotación de recursos faunísticos en el sitio Punta Santa Ana 1: Estrategias de subsistencia de grupos de cazadores marinos tempranos de Patagonia meridional. Magallania 38(1):183-198.

2011. La explotación de recursos faunísticos en el sitio Punta Santa Ana 1: estrategias de subsistencia de grupos de cazadores marinos tempranos de Patagonia meridional. Memoria de Título de arqueólogo, Departamento de Antropología, Universidad de Chile, Santiago. MS.

SAN ROMÁN M., SALAS K. Y FONTUGNE M. 2009. Primeros avances en la reconstrucción de secuencias de ocupación de cazadores recolectores marinos en el estrecho de Magallanes, Patagonia Meridional. En: Arqueología de la Patagonia. Una mirada desde el último confín. Editado por M. Salemme, F. Santiago, M. Álvarez, E. Piana, M. Vázquez y M.E. Mansur, pp. 35-46. Editorial Utopías, Ushuaia.

SIELFELD W., G. GUZMAN Y N. AMADO 2006. Distribución de peces del litoral rocoso de los canales patagónicos occidentales ( $48^{\circ} 37^{\prime} S-53^{\circ} 34^{\prime}$ 'S). Anales del Instituto de la Patagonia 34: 21-32.

TORRES J. 2010. Les stratégies de pêche chez les Indiens canoeros de l'extrémité australe de l'Amérique, pendant la période 2500-3500 BP: Une évaluation depuis l'île Offing, île Dawson et Punta Santa Ana, Détroit de Magellan Patagonie Chilienne. Mémoire Master 2 Archéologie, Université Paris I Panthéon-Sorbonne, Paris. MS.

VARGAS, L. 2008. Peces en Piedra Azul, algo más que huesos de peces. Memoria de Título de arqueólogo, Departamento de Antropología, Universidad de Chile, Santiago. MS.

VENEGAS, C. Y W. SIELFELD 1998. Catálogo de los vertebrados de la región de Magallanes y Antártica Chilena. Ediciones de la Universidad de Magallanes, Punta Arenas.

WHEELER, A. y A. JONES 1989. Fishes. Cambridge University Press, Cambridge.

WÖHLER, O.C.; CASSIA, M.C.; HANSEN, J.E. 2004. Biología y pesquería del bacalao austral (Salilota australis). En: El Mar Argentino y sus recursos pesqueros, Sánchez, R.P. y Bezzi, S.I. editores. Tomo 4. Los peces marinos de interés pesquero. Caracterización biológica y evaluación del estado de explotación. pp.347-359. Publicaciones Especiales INIDEP, Mar del Plata.

ZANGRANDO A. F. 2009a. Historia evolutiva y subsistencia de cazadores recolectores Marítimos de Tierra del Fuego. Sociedad Argentina de Antropología, Buenos Aires. $2009 \mathrm{~b}$. Is fishing intensification a direct route to huntergatherer complexity? A case study from the Beagle Channel region (Tierra del Fuego, southern South America). World Archaeology 41(4):589-608 